\title{
Yield of screening blood work and MRI of the brain and orbits in the work-up of unilateral chronic optic neuropathy
}

\author{
Elie Côté ${ }^{1}{ }^{1} \cdot$ Ari Aharon Shemesh ${ }^{1} \cdot$ Lili Tong $^{1} \cdot$ Cindy T. Y. Lam ${ }^{1} \cdot$ Edward Margolin ${ }^{1}$
}

Received: 26 April 2018 / Revised: 3 September 2018 / Accepted: 1 October 2018 / Published online: 13 February 2019

(c) The Royal College of Ophthalmologists 2019

\begin{abstract}
Background/Objectives No guidelines exist for the investigation of treatable causes of chronic optic neuropathy, including sarcoidosis, lupus, and syphilis. The purpose of this study was to determine the diagnostic yield of screening blood work (ACE (Angiotensin Converting Enzyme) for sarcoidosis, Antinuclear Antibodies (ANA) for lupus, CMIA (chemiluminescence microparticle enzyme immunoassay) for syphilis) and contrast-enhanced MRI brain and orbits in atypical unilateral chronic optic neuropathy.

Subjects/Methods Retrospective review from February 2012 to June 2018 at a neuro-ophthalmology practice. Six hundred and eighty-three consecutive charts with optic neuropathy were reviewed. Inclusion criteria were unilateral chronic optic neuropathy and a work-up including contrast-enhanced MRI brain and orbits, CBC, ESR, CRP, ANA, CMIA, and ACE. Exclusion criteria were optic nerve swelling in either eye on initial assessment or an established cause of optic neuropathy. The main outcome measure was diagnostic yield.

Results Fifty-seven patients were included. One patient had elevated ACE, seven had positive ANA titers, and three had positive CMIA. Zero patients were diagnosed with sarcoidosis, one patient was diagnosed with lupus-related optic neuropathy, and one patient was diagnosed with syphilitic optic neuropathy. The diagnostic yield of ACE was 0\%, ANA was $1.75 \%$, and CMIA was $1.75 \%$. MRI revealed planum sphenoidale meningioma causing compressive optic neuropathy in one patient, giving it a diagnostic yield of $1.82 \%$.

Conclusion Routine screening blood work (ACE, ANA, CMIA) and MRI brain and orbits for chronic idiopathic unilateral optic neuropathy has low diagnostic yield, especially if clinical suspicion for syphilis, lupus, and sarcoidosis is low. MRI should still be performed in all cases in order to rule out compressive lesions.
\end{abstract}

\section{Introduction}

The most common cause of non-glaucomatous optic neuropathy in patients over the age of 50 is anterior ischemic optic neuropathy and guidelines for investigations of this condition are well described [1]. In many cases though the etiology of the chronic optic neuropathy is unclear, as patients present with the absence of typical features pinpointing to a specific cause of optic neuropathy (e.g. typical features present in non-arteritic anterior ischemic optic neuropathy (NAION) such as disc at risk in

Edward Margolin

edmargolin@gmail.com

1 Department of Ophthalmology \& Vision Sciences, University of Toronto, Toronto, ON, Canada the other eye, acute onset of visual loss, altitudinal visual field defect, etc.). In these cases without an obvious etiology of optic neuropathy, the workup is often broadened and includes additional screening blood work for potentially treatable causes of chronic optic neuropathy such as sarcoidosis, lupus, and syphilis-related optic neuropathy despite these conditions being uncommon $[2,3]$. There are no guidelines for the investigative approach of these uncommon causes of chronic optic neuropathy. Furthermore, there is a lack of evidence regarding the diagnostic yield of these tests, especially in cases where clinical suspicion is low, and it is possible that these tests may not be necessary. In cases of high pretest probability a negative result may not be useful, especially if a test has poor diagnostic accuracy. The purpose of this study was to determine the diagnostic yield of screening blood tests (ACE for sarcoidosis, ANA for lupus, CMIA for syphilis) and contrast-enhanced 
magnetic resonance imaging (MRI) of the brain and orbits in atypical cases of idiopathic unilateral chronic optic neuropathy.

\section{Methods}

A retrospective consecutive chart review of patients with a diagnosis of chronic unilateral optic neuropathy seen in a tertiary neuro-ophthalmology practice between February 2012 and June 2018 was carried out. All patients were identified using the diagnostic codes for optic neuropathy. This was verified by reviewing all the charts that were identified using this code to determine if they fulfilled inclusion criteria. Patients were included if they had a unilateral chronic optic neuropathy diagnosed at the first clinical visit, lack of established and common etiologies of their optic neuropathy, and had an optic neuropathy workup including MRI of the brain and orbits with gadolinium (or computed tomography (CT) brain and orbits if they had metal implants/pacemaker), complete blood count (CBC), erythrocyte sedimentation rate (ESR), C-reactive protein (CRP), ANA, CMIA and ACE. Chronic optic neuropathy was defined as pallor of the optic nerve head and at least 1 month duration of visual loss. Patients were excluded if they had optic nerve swelling in either eye on initial assessment or an obvious established cause of their optic neuropathy.

Established causes included patients with previously diagnosed ocular or systemic conditions that would cause an optic neuropathy, or patients who had a history and physical exam findings typical for the following conditions: demyelinating optic neuritis (patients with a history of subacute visual loss accompanied by pain on eye movements and subsequent resolution of visual deficits within 1-3 months), NAION (patients over 50 years of age with disc-at-risk in the fellow eye with a history of sudden painless vision loss, and presence of altitudinal visual defect), and arteritic ischemic optic neuropathy (history of systemic symptoms of giant cell arteritis accompanied by severe vision loss, and/or positive temporal artery biopsy). Thus, only patients with atypical presentations of chronic optic neuropathies not fitting a clear diagnosis were included.

Patients with reactive CMIA had further testing with RPR and TPPA to confirm the diagnosis, as per the standard procedure of the Ontario Public Health Laboratory where samples were sent. Patients with positive ACE had contrastenhanced MRI of the brain and orbits for neurosarcoidosis that was reviewed individually with a neuro-radiologist and CT of the thorax for pulmonary sarcoidosis. Patients with elevated ANA titers were referred to a rheumatologist if they had one of the following: ANA titers $\geq 1: 640$ or elevated ANA titers of any level with other signs and symptoms of lupus. ANA result was considered false positive in patients with ANA titerss of $\geq 1: 640$ who were referred to a rheumatologist and extensive workup failed to support diagnosis of systemic lupus erythematosus (SLE) and in patients with lower ANA titers $(\leq 1: 320)$ who had no other signs/symptoms of lupus and thus were deemed not to have a diagnosis of SLE as well.

Data collected included age, gender, duration of symptoms, result of blood tests, results of neuroimaging, and final diagnosis. Diagnostic yield (\% of cases where blood work or neuroimaging established the diagnosis) and positive predictive value (PPV) were calculated for each test. This study was approved by the Research Ethics Board of the University of Toronto.

\section{Results}

A total of 683 consecutive charts of patients with a diagnosis of optic neuropathy were reviewed. Fifty-seven patients with chronic unilateral optic neuropathy assessed by a single neuro-ophthalmologist met inclusion criteria. Twenty-three $(40.4 \%)$ of the patients were male. The average age at presentation was $61.2 \pm 16.7$ years. Seventeen $(29.8 \%)$ patients had greater than 1 year of vision loss, and $21(36.8 \%)$ patients were asymptomatic (Fig. 1). Figure 2 displays the final diagnosis of undifferentiated chronic optic neuropathy in our study. The most common causes were presumed NAION $(N=24)$ and optic neuropathy NYD $(N=24)$. Inflammatory and infectious etiologies were uncommon $(N=1$ and 1 , respectively). Patients were given a diagnosis of optic neuropathy NYD if they lacked typical features of NAION (e.g. no disc-at-risk in the fellow eye) or had an atypical feature (e.g. worsening of visual acuity beyond 2 weeks after onset of visual loss) and if they did not fit the typical pattern seen in demyelinating optic neuritis (subacute visual loss accompanied by pain on eye movements with resolution of visual deficits within 1-3 months) and workup for other causes was negative.

One patient had elevated ACE level, seven patients had positive ANA titers, and three patients had positive CMIA

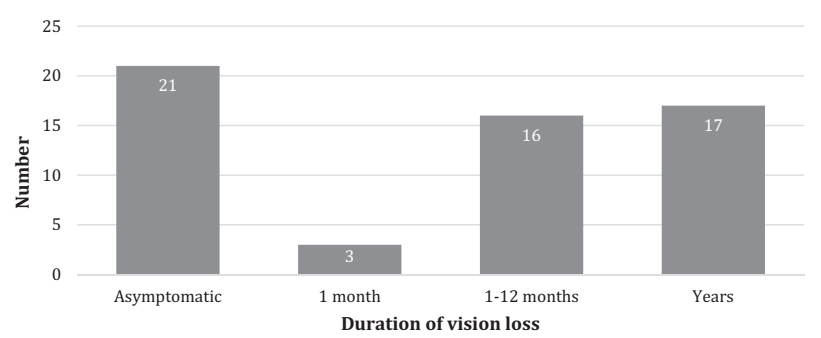

Fig. 1 Duration of vision loss in patients presenting with atypical unilateral chronic optic neuropathy 
screen. Following further investigations, no patients were diagnosed with sarcoidosis, one patient with a high ANA titer (>1:320) with a maternal history of lupus was diagnosed with lupus-related optic neuropathy, and one patient with reactive CMIA was diagnosed with syphilitic optic neuropathy.

The patient who was diagnosed with syphilitic optic neuropathy presented with a pale optic nerve in one eye and was found to have positive CMIA and subsequently positive TPPA but negative RPR. He was previously treated for gonorrhea. Upon reviewing the patient's history and laboratory results, an infectious disease consultant had decided that the patient most likely had untreated secondary syphilis and he was treated with intravenous penicillin injections.

The patient who was diagnosed with lupus-related optic neuropathy presented with a 2-year history of semi-acute painless visual loss in the right eye. On exam, central acuity was 20/400 in the affected eye, there was an obvious relative afferent pupillary defect (RAPD), and the affected optic nerve was diffusely pale. On review of systems, she had a recent episode of acute onset ankle swelling with pain and inability to weight bear (the ankle was still swollen at our clinic), and an episode of knee swelling with pain that lasted over a month. She also recalled two episodes of acute onset chest pain lasting a few days, relieved with positioning, which was felt to be likely secondary to pericarditis. MRI of the brain demonstrated smaller right optic nerve but no other abnormalities. ANA titer was 1:320, and the rest of the extensive rheumatologic workup was negative. Based on the presence of positive ANA titers, history of oligoarthritis and pericarditis, as well as optic neuropathy, the

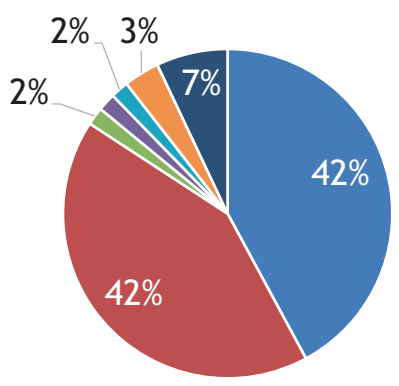

- NAION
- Optic Neuropathy NYD
- Compressive lesion
- Inflammatory
- Infectious
- Anomalous Nerve
- Other

Fig. 2 Final diagnosis of cases of undifferentiated unilateral chronic optic neuropathy

Table 1 Diagnostic yield and positive predictive value of ACE, ANA, and CMIA diagnosis of SLE was made and hydroxychloroquine therapy was initiated.

The other two CMIA reactive patients had remotely treated syphilis, which was felt to be unrelated to their optic neuropathy. The patient with increased serum ACE levels had undergone further sarcoidosis workup, including contrast-enhanced MRI of the brain and orbits for neurosarcoidosis that was reviewed individually with a neuroradiologist and CT of the thorax for pulmonary sarcoidosis, which were both negative. The remaining six patients with positive ANA results were investigated to rule out connective tissue disease (CTD). Two patients were referred to a rheumatologist for further workup: one patient with very high ANA titers $(>1: 640)$ and the other patient for suspicious vasculitic lesions on MRI. In both cases, lupus and other CTD were ruled out after extensive rheumatological work-up. The remaining four patients had lower ANA titers: one patient with a titer of 1:320, two patients with a titer of $1: 160$, and one patient with a titer of $1: 80$, all of which exhibited nucleolar pattern. SLE was excluded in these patients based on lack of other signs or symptoms of lupus, lack of family history of lupus, and the fact that nucleolar pattern is less commonly associated with SLE than other staining patterns. Full workup for connective tissue disorders was carried out in all patients by a rheumatologist. All patients with ANA titers of over 1:80 had NeuroMyelitis Optica antibody testing performed as well and it was negative in all.

All patients had contrast-enhanced MRI of the brain and orbits, except for two patients who had CT brain and orbits due to having a pacemaker. MRI was diagnostic in one patient revealing large planum sphenoidale meningioma causing compressive optic neuropathy. In the remaining patients, MRI was either non-contributory (e.g. demonstrated non-specific white matter changes) or normal. CT scan was normal or non-contributory in both patients who had it instead of an MRI. CBC, ESR, and CRP were normal in all patients with positive ACE, ANA, or CMIA blood tests.

Overall, the diagnostic yield of ACE was 0\%, ANA was $1.75 \%$, and CMIA was $1.75 \%$. The diagnostic yield of MRI was $1.82 \%$. The overall diagnostic yield of neuroimaging (MRI or CT) was $1.75 \%$. The PPV of ACE, ANA, and CMIA were $0 \%, 14.3 \%$, and $100 \%$, respectively (Table 1 ).

\begin{tabular}{llllll}
\hline Test & $\begin{array}{l}\text { Total patients } \\
\text { tested }(N)\end{array}$ & $\begin{array}{l}\text { Positive test } \\
(N)\end{array}$ & $\begin{array}{l}\text { Disease positive } \\
(N)\end{array}$ & $\begin{array}{l}\text { Positive predictive } \\
\text { value }(\%)\end{array}$ & $\begin{array}{l}\text { Diagnostic yield } \\
(\%)\end{array}$ \\
\hline ACE & 57 & 1 & 0 & 0 & 0 \\
ANA & 57 & 7 & 1 & 14.3 & 1.75 \\
CMIA & 57 & 3 & $3^{\text {a }}$ & 100 & 1.75 \\
\hline
\end{tabular}

ane patient with positive CMIA diagnosed with syphilitic optic neuropathy. The other two CMIA-positive patients had remote treated syphilis unrelated to optic neuropathy 


\section{Discussion}

Investigating patients with atypical chronic optic neuropathy can be challenging for the clinician and no guidelines exist for the diagnostic workup of patients with this condition. As a result, these patients are often investigated with screening blood work, even if the clinical suspicion of a disease that is being screened for is low, as potentially treatable causes may be identified. There is limited literature describing the diagnostic yield of additional screening blood tests in these patients and evidence is needed to help guide clinicians in these scenarios. This study reports the diagnostic yield of ACE, ANA, CMIA, and MRI of the brain and orbits with contrast in the workup of patients with atypical unilateral chronic optic neuropathy.

All patients presenting with a pale optic nerve should have investigations tailored to their history and examination findings. Most clinicians rely on pattern recognition when evaluating patients presenting with a pale optic nerve in one or both eyes. Patients fitting a pattern seen in demyelinating optic neuritis, NAION, AION as well as patients who have bilateral symmetric optic neuropathy without the presence of RAPD (i.e. typical for inherited causes of optic neuropathy) do not require screening testing but instead should have diagnostic investigations tailored to their history and exam findings. All of these patients were excluded from our study. Only those patients who did not have history and/or physician examination findings typical for these conditions were further investigated with blood tests for infectious and non-infectious inflammatory causes (CBC, ESR, CRP, ANA, ACE, CMIA) as well as neuroimaging (contrastenhanced MRI of the brain and orbits).

Limited literature on this subject suggests performing neuroimaging and blood tests tailored to the clinical suspicion. Singh et al. [4] suggested that first-line investigations including CBC, ESR, CMIA, and MRI of the brain and orbits should be performed in all patients and more specific testing (ACE and ANA) only if the clinical suspicion is high. One previous study suggested that screening investigations have low yield in the evaluation of unexplained optic atrophy: Lee et al. [5] retrospectively reviewed the yield of any laboratory testing (blood work, lumbar puncture, carotid Doppler, and heavy metal panel) as screening tests for 51 patients with unexplained optic atrophy, and found a diagnostic yield of $0 \%$. Similarly, in our study, we found a low diagnostic yield of $0 \%, 1.75 \%$, and $1.75 \%$ for ACE, ANA, and CMIA, respectively, in evaluating patients with chronic unilateral optic neuropathy. This suggests that ordering these blood tests in all cases may be unnecessary and should be reserved for cases where clinical suspicion for any particular disease that is being screened for is high.
Interestingly, Lee et al. [5] found that neuroimaging provided an etiology of optic neuropathy in $20 \%$ of patients with unexplained optic atrophy. In our study MRI had a low diagnostic yield of $1.82 \%$. This may be partly explained by the fact that the majority of patients with neuroimaging findings elucidating the cause of optic neuropathy in the study by Lee et al. had bilateral optic atrophy. We only included patients with unilateral optic neuropathy in our study excluding all patients who had bilateral optic nerve pallor. Despite the low yield of contrast-enhanced MRI of the brain and orbits in our study, it is still warranted in investigation of unilateral optic neuropathy in all patients who do not have a classic clinical picture consistent with prior NAION or prior episode of demyelinating optic neuritis, and especially in cases of progressive visual loss, in order to exclude the presence of structural lesions.

Optic neuropathy occurs in only $1 \%$ of lupus patients and lupus presenting as optic neuropathy is very rare [6]. Most patients with lupus-associated optic neuropathy have other signs or symptoms of CTD [6]. In our study, screening with ANA led to one diagnosis of lupus optic neuropathy, giving it a diagnostic yield of $1.75 \%$ in patients with unilateral chronic optic neuropathy. Three patients had ANA titers greater than 1:320; there were six patients with falsepositive ANA tests. In one patient who was ultimately diagnosed with SLE, clinical suspicion of lupus was high given a strongly positive family history. In six patients with false-positive ANA tests, pre-test clinical suspicion was low, as the patients had no other signs or symptoms suggestive of lupus. ANA is known to have poor diagnostic accuracy with a reported PPV of $29 \%$ [7]. We found an even lower PPV of $14.3 \%$ in the context of chronic unilateral optic neuropathy. Moreover, $20 \%$ of healthy individuals have a positive ANA titers of 1:40, and 5\% of healthy individuals have a clinically significant titers of 1:160 [7]. False positives may lead to patient anxiety as well as unnecessary follow-up investigations. Additionally, ANA is often elevated in other conditions including other CTDs and certain infections. As a result, medical societies including the Canadian Rheumatologic Association recommend against ANA as a screening blood test in a patient with no signs or symptoms of lupus [7].

Syphilis screen is also often included in the idiopathic chronic optic neuropathy workup even if clinical suspicion is low. Although syphilis may present with isolated ocular involvement, other signs and symptoms of syphilis are often present as well [8]. Syphilis has four disease stages: primary, secondary, latent, and late. Ocular involvement occurs most commonly during the secondary and later stages [8]. Reactive CMIA in our study led to one diagnosis of syphilitic optic neuropathy with the diagnostic yield of $1.75 \%$. In this one patient pre-test clinical suspicion for syphilis was high as there was a positive history of recent 
other sexually transmitted infection (STI). The other two cases of positive CMIA were in patients who had been remotely treated for syphilis and thus believed to be unrelated to their optic neuropathy. In the remaining 54 patients with low clinical suspicion, CMIA was negative and of low diagnostic value. CMIA has been reported to have very high sensitivity of $99-100 \%$, which is ideal for a screening test [9]. Furthermore, it also has a high PPV of 91.47\% [9]. Similarly, we found a PPV of CMIA of $100 \%$ in our study. Therefore, CMIA screening may be useful in the workup of chronic optic neuropathy especially if the pre-test clinical suspicion is high as evidenced by history of STIs or other manifestations of syphilis.

Neurosarcoidosis, which includes optic neuropathy, is the presenting manifestation of sarcoidosis in only $1 \%$ of cases [2]. Although serum ACE is very commonly performed as a screening blood test in patients with idiopathic chronic optic neuropathy, it is known to be an insensitive test in diagnosing neurosarcoidosis. In our study, ACE was positive in one patient, who was not ultimately diagnosed with sarcoidosis. This translated into a diagnostic yield of $0 \%$ for ACE screening in patients with chronic unilateral optic neuropathies. Serum ACE has been shown to have poor diagnostic accuracy, with a sensitivity ranging from $25 \%$ to $45 \%$ in patients with biopsy-proven sarcoid optic neuropathy [2, 10]. Furthermore, Ungprasert et al. [11] reported a PPV of only $25.4 \%$ for ACE in sarcoid patients. We report an even lower PPV of $0 \%$ of serum ACE testing in patients with undifferentiated unilateral chronic optic neuropathy. Although serum ACE levels are increased in approximately half of patients with sarcoidosis, it may also be elevated in other granulomatous diseases as well as in other conditions such as diabetes mellitus [2]. Therefore, ACE levels have both a low value as a screening blood test and as a diagnostic test. In cases of high clinical suspicion more sensitive testing for neurosarcoidosis such as an MRI of the brain and orbits with gadolinium, gallium scan, and potentially positron emission tomography should be performed [12].

Our study is limited by selection bias given its retrospective design. It is also possible that our database search did not identify all cases of chronic unilateral optic neuropathy as patients with multiple ocular co-morbidities might have been labeled with a different diagnostic code. We also excluded all patients with chronic unilateral optic neuropathy who did not receive screening blood work for ANA, CMIA, and ACE (cases where the cause of optic neuropathy was established based on the history and exam findings, such as demyelinating optic neuritis, NAION, and AION). Lastly, the patients in our study were drawn from a tertiary/quaternary neuro-ophthalmology practice creating a sampling bias. Patients with optic neuropathy seen in the community may have received an appropriate workup and diagnosis avoiding a referral to our center. As a result, we may have included more complex cases of chronic unilateral optic neuropathy in which no etiology was previously identified. Therefore, these results may not be generalizable to all ophthalmology practices. The combination of these factors (selection bias, case identification, and patients avoiding referral to our center) may have artificially lowered the prevalence of patients seen with lupus, sarcoidosis, and syphilis in our study compared to the general population. Thus, our low PPV values for lupus and sarcoidosis may be partly a result of this factor. Similarly, patients with MRI findings explaining the presence of optic neuropathy may have already been identified in outside centers and not referred to us as diagnosis was already made.

In conclusion, our study demonstrates that routine screening blood work (ANA titers, ACE level, and CMIA screening) in patients with idiopathic chronic unilateral optic neuropathy has low diagnostic yield. Therefore, these screening tests should only be ordered after careful consideration of a patient's individual presentation and only if clinical suspicion is high. While contrast-enhanced MRI of the brain and orbits had a low diagnostic yield in our study, it is still of paramount importance in all patients with chronic optic neuropathy in order to exclude structural lesions. These results highlight that sometimes extensive workup is undertaken in order to attempt to diagnose a treatable condition even though the yield of this workup may be very low.

\section{Summary}

\section{What was known before}

- No guidelines exist for the investigation of treatable causes of idiopathic chronic optic neuropathy, such as sarcoidosis, lupus, and syphilis.

\section{What this study adds}

- Routine screening blood work (ANA titers, ACE level, and CMIA screen) for idiopathic chronic optic neuropathy has low diagnostic yield - these blood tests should only be ordered if clinical suspicion is high.

\section{Compliance with ethical standards}

Conflict of interest The authors declare that they have no conflict of interest.

Publisher's note: Springer Nature remains neutral with regard to jurisdictional claims in published maps and institutional affiliations. 


\section{References}

1. Biousse V, Newman NJ. Ischemic optic neuropathies. N Engl J Med. 2015;372:2428-36.

2. Boudreault K, Durand ML, Rizzo JF. Investigation-directed approach to inflammatory optic neuropathies. Semin Ophthalmol. 2016;31:117-30.

3. Voss E, Raab P, Trebst C, Stangel M. Clinical approach to optic neuritis: pitfalls, red flags and differential diagnosis. Ther Adv Neurol Disord. 2011;4:123-34.

4. Singh Digvijay, Saxena Rohit, Sharma Pradeep, Menon Vimla. Systematic approach to a case of disc pallor. Delhi J Ophthalmol. 2011;21:28-32.

5. Lee AG, Chau FY, Golnik KC, Kardon RH, Wall M. The diagnostic yield of the evaluation for isolated unexplained optic atrophy. Ophthalmology. 2005;112:757-9.

6. Frigui M, Frikha F, Sellemi D, Chouayakh F, Feki J, Bahloul Z. Optic neuropathy as a presenting feature of systemic lupus erythematosus: two case reports and literature review. Lupus. 2011;20:1214-8.
7. Chow SL, Carter Thorne J, Bell MJ, Ferrari R, Bagheri Z, Boyd T, et al. Choosing wisely: the Canadian Rheumatology Association's list of 5 items physicians and patients should question. J Rheumatol. 2015;42:682-9.

8. Durnian JM, Naylor G, Saeed AM. Ocular syphilis: the return of an old acquaintance. Eye (Lond). 2004;18:440-2.

9. Zhou J, Liang Y, Zhang J, Cui L. The analyzation and clinical evaluation of ECLIA and CMIA in the detection of Treponema pallidum. Medicine (Baltimore). 2017;96:e7139.

10. Koczman JJ, Rouleau J, Gaunt M, Kardon RH, Wall M, Lee AG. Neuro-ophthalmic sarcoidosis: the University of Iowa experience. Semin Ophthalmol. 2008;23:157-68.

11. Ungprasert P, Carmona EM, Crowson CS, Matteson EL. Diagnostic utility of angiotensin-converting enzyme in sarcoidosis: a population-based study. Lung. 2016;194: 91-5.

12. Ibitoye RT, Wilkins A, Scolding NJ. Neurosarcoidosis: a clinical approach to diagnosis and management. J Neurol. 2017;264: $1023-8$. 